\title{
Revegetación de suelo arenizado con Butia lallemantii en el suroeste del estado de Rio Grande do Sul, Brasil
}

\author{
Revegetation of sandblasted soil with Butia lallemantii in the southwestern state \\ of Rio Grande do Sul, Brazil
}

\author{
Luciana Pinto Paim a*, Eduarda Demari Avrella a, Elisete Maria de Freitas ${ }^{\text {b }}$, Claudimar Sidnei Fior a \\ *Autor de correspondencia: a Universidade Federal do Rio Grande do Sul, Faculdade de Agronomia, \\ Departamento de Horticultura e Silvicultura, Avenida Bento Gonçalves, 7712, Porto Alegre, Rio Grande do Sul, \\ Brasil, tel.: +55 051 996885515, lucianappaim@bol.com.br \\ b Universidade do Vale do Taquari, Programa de Pós-graduação em Sistemas Ambientais Sustentáveis, \\ Programa de Pós-graduação em Biotecnologia, Lajeado, Rio Grande do Sul, Brasil.
}

\begin{abstract}
SUMMARY
Butia lallemantii is endemic from sandy soils in the state of Rio Grande do Sul (RS). Sandblasted soils stand out for fragile characteristics of low fertility, water availability and intense sand displacement. However, studies on planting techniques, use of fertilizers and alternative products that may favor the establishment of plants in these soils are lacking. Therefore, the objective of the study was to evaluate the survival and leaf appearance of the B. lallemantii plants established by the revegetation method in sandy soil, with the increase of mineral fertilizer and hydroretentor polymer, as well as monitoring the movement of sand particles. The plants were collected in Alegrete / RS, classified in stipe diameter and kept in substrate (soil, animal waste and rice husk, 1:1:1) for eight months. In field plantation, the doses of NPK and hydrogel were randomly placed between five graded rules in the soil, for periodic sand displacement readings. Survival, appearance of live leaves and sand displacement were evaluated monthly, with a randomized block design (100 seedlings/block). Results showed higher survival and presence of living leaves in the plants of block one, and average displacement of sand in the spring period. Therefore, plants of larger stipe diameter class show higher potential for survival and larger number of live leaves, in addition, sand displacement mainly happens during spring.
\end{abstract}

Key words: Pampa biome, sand displacement, recovery, fragile soils.

\section{RESUMEN}

Butia lallemantii es endémica proveniente de suelos arenosos y arenizados en el estado de Rio Grande do Sul (RS). Los suelos arenizados se destacan por características frágiles de baja fertilidad, disponibilidad de agua y por intenso desplazamiento de arena. Sin embargo, faltan estudios acerca de técnicas de plantación, uso de fertilizantes y productos alternativos que pueden favorecer el establecimiento de plantas en estos suelos. Por lo tanto, el objetivo del estudio fue evaluar la supervivencia y el aspecto foliar de las plantas de $B$. lallemantii establecidas por el método de revegetación en el suelo arenizado, con el incremento de fertilizante mineral y polímero hidroretentor, así como monitorear el desplazamiento de partículas de arena. Las plantas fueron recolectadas en Alegrete/ RS, clasificadas de diámetro del estípite y se mantuvieron en sustrato (suelo, residuos animales y cáscara de arroz, 1:1:1), durante ocho meses. En plantación de campo, las dosis de NPK e hidrogel fueron colocadas aleatoriamente entre cinco reglas graduadas en el suelo, para lecturas periódicas de desplazamiento de arena. La supervivencia, la apariencia de hojas vivas y el desplazamiento de la arena se evaluaron mensualmente, con diseño en bloques al azar (100 plántulas/bloque). Los resultados mostraron una mayor supervivencia y presencia de hojas vivas en las plantas de bloque uno, y un desplazamiento promedio de arena en el período de primavera. Por lo tanto, las plantas de mayor clase de diámetro del estípite pose en alto potencial de supervivencia y de hojas vivas, además, la temporada de primavera mayor desplazamiento de arena.

Palabras clave: bioma Pampa, desplazamiento de arena, recuperación, suelos frágiles.

\section{INTRODUCCIÓN}

En Rio Grande do Sul (RS), el bioma Pampa engloba más de la mitad del estado del Sur (Boldrini et al. 2010), abarcando el $63 \%$ del territorio e innumerables áreas para el desarrollo de ganadería extensiva (Pillar et al. 2009). $\mathrm{Al}$ suroeste del estado del RS, se evidencia un paisaje con suelos de textura arenosa, derivados de formaciones areníticas denominadas Formaciones Guará y Botucatu, siendo locales caracterizados como fenómeno de arenización 
(Trindade et al. 2008, Vieira y Verdum 2015). Estas áreas son intensificadas por acciones antrópicas (como superpastoreo) y prácticas agrícolas, bien como por sus factores edafoclimáticos de la región, como suelo arenizado, lluvias y vientos acentuados, los cuales actúan de forma erosiva en el transporte de sedimentos sobre la superficie vulnerable del suelo arenizado (Binda y Verdum 2015, Rovedder y Eltz 2008a).

Estos lugares son conocidos regionalmente como arenales, presentando pérdida de la cobertura vegetal por la intensa acción de procesos erosivos hídricos y eólicos, sobretodo el eólico, cuyo desplazamiento de partículas de arena ocasiona efecto abrasivo y soterramiento de las plantas, consecuentemente, exposición del suelo arenoso (Rovedder y Eltz 2008b, Rovedder et al. 2010). Estos suelos recientes (Entisol: Quartzipsamments) pocos profundos golpeados por el proceso de arenización presentan $\mathrm{pH}$ ácido, con exceso de aluminio y escasez de fósforo y potasio, siendo que la precariedad del fósforo en esos suelos resultará en disminución de procesos energéticos del metabolismo vegetal, limitando el crecimiento vegetativo, la floración y la formación de las plantas (Suertegaray 2011, Suertegaray y Oliveira 2014).

En la recuperación de áreas degradadas, el método de revegetación del suelo es una importante técnica cuyo objetivo promueve el retorno de las condiciones deseables al ambiente, mediante del uso de especies con características de acelerado y eficiente desarrollo en áreas fragilizadas (Rovedder et al. 2010, Schneider et al. 2014). En este contexto, la especie de Butia lallemantii Deble et Marchiori presenta características relevantes para la recuperación de esas áreas dañadas, debido su ocurrencia natural y exclusiva en campos de suelos arenosos y en las elevaciones areníticas de diversos municipios del suroeste del RS (Soares et al. 2014). Además, la fructificación de esta especie ocurre por todo el año y citan como características morfológicas de esos individuos, el porte bajo, las pinas estrechas, además de la presencia de estípite cespitoso (Soares et al. 2014). El hábito cespitoso de esa palmera, formando un grupo, permite la separación de plantas como forma de propagación, cuando presentan condiciones vigorosas (Paim y Paim 2016).

Algunos recursos utilizados en plantíos comerciales de especies hortícolas y forestales han ayudado en el establecimiento de plantas, principalmente en regiones donde el estrés hídrico ocurre en la época de plantación. En el caso de los hidroretentor, es necesario tener en cuenta que el uso de polímeros sintéticos derivados del petróleo, con propiedades físico-químicas de absorber agua y nutrientes solubles, los cuales se liberan conforme la disminución del potencial hídrico del ambiente, así como del hidrogel (Bernardi et al. 2012). En la mayoría de los casos, el hidrogel actúa como un polímero hidrofílico superabsorbente, aumentando el volumen de retención de agua, tanto en suelos y en sustratos (Vieira y Pauletto 2009). De este modo, la aplicación de polímeros hidrorententores en la plantación de plantas puede favorecer el estado nutricional de las plantas, con períodos prolongados de disponibilidad de solución de nutrientes, reducción del proceso de lixiviación en el suelo, capacidad de cambio de cationes del polímero y de taponamiento del suelo $\mathrm{pH}$ (Bernardi et al. 2012).

Otro factor importante es el empleo de la fertilización, el cual está directamente asociado a la calidad de las plantas, pues ejerce una influencia notable en el sistema radicular y en el estado nutricional de las plantas, principalmente en el caso de especies nativas que aún es poco conocido (Tucci et al. 2009, Rodrigues et al. 2010). La restricción de nutrientes ha sido uno de los aspectos responsables por la pérdida de plantas y altas mortalidades de las plantas en el momento de la plantación a campo, ya que los niveles nutricionales tienen gran interferencia en la calidad de las plantas y en el desarrollo del campo, especialmente los elementos esenciales como nitrógeno, fósforo y potasio (Tucci et al. 2009).

A base de esto, en el suroeste de RS, los suelos afectados por el fenómeno de arenización presentan áreas improductivas para el cultivo agrícola y la expansión del ganado, siendo prácticas características del desarrollo económico de esta región. Por lo tanto, es imprescindible realizar estudios centrados en el manejo de técnicas eficientes para la revegetación de estos suelos arenizados, a fin de reducir los efectos negativos de su baja fertilidad y retención de agua, así como los procesos de erosión hídrica y eólica, para beneficiar la supervivencia de las especies en estos lugares. De ese modo, este estudio tiene como hipótesis que la incorporación de las técnicas que utilizan fertilizantes minerales y el polímero hidroretentor pueden favorecer el establecimiento y la supervivencia de las plantas de $B$. lallemantii en suelos arenizados desprovistos de esta especie. El objetivo principal de este trabajo es estudiar la supervivencia y el aspecto foliar de las plantas de B. lallemantii, que fueran establecidas por el método de revegetación en un suelo arenizado desprovisto de esta especie, mediante el uso de fertilizantes minerales NPK (5-30-15) y polímero de hidroretentor (hidrogel), así como monitorear el desplazamiento de partículas de arena en áreas adyacentes a individuos de $B$. lallemantii.

\section{MÉTODOS}

Caracterización del área de estudio. El presente estudio fue realizado en la propiedad rural Duas Guias, la cual está situada en el municipio de Alegrete/RS (29 $47^{\prime} 48^{\prime \prime} \mathrm{S}$, $55^{\circ} 24^{\prime} 20^{\prime \prime} \mathrm{W}$ ), con aproximadamente 94 ha, presentando actualmente, aproximadamente a 19 ha alcanzados por el proceso de arenización. El área experimental fue instalada en el área arenizada, con tamaño de 0,5 ha y declive en torno al $3 \%$, siendo aislada con valla electrificada para impedir el acceso de animales herbívoros de la propiedad. El clima de la región se caracteriza como subtropical medianamente húmedo a húmedo, con lluvias anuales de 1.500 a $1.800 \mathrm{~mm}$, bien distribuidas durante el período de 
90 a 120 días lluviosos, durante todo el año. La temperatura media anual evidencia variación entre 17 a $20^{\circ} \mathrm{C}$, siendo que la temperatura media del mes más frío oscila entre 11 a $14{ }^{\circ} \mathrm{C}$ y la del mes más caliente varía entre 23 a $26{ }^{\circ} \mathrm{C}$ (Rossato 2011).

Establecimiento de las plantas de B. lallemantii en el área de estudio. Las plantas de B. lallemantii (figura 1) fueron recolectadas en ambiente de campo nativo bajo intensa disponibilidad de luz, en el período de mayo de 2015, en la Hacienda Tres Nascentes, ubicada en Alegrete/RS

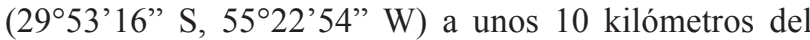
área de estudio. Las plantas se obtuvieron agrupando seis grupos de plantas con un diámetro medio de $1,0 \mathrm{~m}$ cada uno, contabilizando, en promedio, 65 plantas de raíces desnudas en cada grupo de plantas, las cuales estaban situadas en una población con densidad media de 40 grupos por hectárea. El procedimiento de recolección fue conforme al método de Paim y Paim (2016), el cual se realizó la remoción del suelo cuidadosamente a una distancia de 30 a $50 \mathrm{~cm}$ alrededor de los grupos de plantas, con uso de utensilios adecuados (palas de corte y azadas), con el fin de evitar daños a las plantas. Las raíces $\mathrm{y}$ hojas de todas las plantas fueron podadas, dejando cerca de $5 \mathrm{~cm}$ de longitud de las raíces y $25 \mathrm{~cm}$ de longitud de la parte aérea desde el estípite. Después de la separación y recuento de los individuos obtenidos en cada grupo de plantas y de acuerdo con el número total de plantas, las mismas fueron clasificadas en tres clases de diámetro del estípite - CDE (pequeñas - CDE 1: menores de 4,2 cm; medianas - CDE 2: 4,21 a 6,4 cm, grandes - CDE 3: 6,41 a más de $9 \mathrm{~cm}$ ).

Después del procedimiento de poda y clasificación, las plantas de $B$. lallemantii se mantuvieron durante ocho meses en macetas de polietileno negro con capacidad de dos litros, llenadas con sustrato compuesto por la mezcla de suelo arenizado (Neossolo Quartzarênico definido taxonómicamente como Entisol: Quartzipsamments), estiércol bovino, cáscara arroz in natura y gris de cáscara de arroz $(1: 1: 1: 1, \mathrm{v} / \mathrm{v})$. Las plantas se mantuvieron en la propiedad cercana al área experimental, bajo la condición de sombreado de otros árboles en el período de la mañana e insolación directa por la tarde, con dos irrigaciones semanales.

En el momento de la plantación, en diciembre de 2015, se aplicaron cinco dosis de abono granulado (formulación NPK 5-30-15) en cantidades de 0, 30, 60, 90 y 120 g por plantín. De este modo, 20 plantas de cada clase de diámetro del estípite fueron abonadas con una de las cinco dosis de NPK. En todas las plantas se realizó aplicación de $40 \mathrm{~g}$ de calcáreo dolomítico (PRNT $65 \%$ ), en superficie acerca de $10 \mathrm{~cm}$ de las plantas. Se aplicó calcáreo para ser una fuente de corrección de $\mathrm{pH}$, ya que se consideran suelos ácidos. Además, se eligió una distancia de $10 \mathrm{~cm}$, porque las plantas fueron podadas, es decir, su proyección de dosel era menor o igual a $10 \mathrm{~cm}$. El espaciamiento utilizado fue de $2 \times 1 \mathrm{~m}$, por cuenta de la ausencia de informaciones sobre el espaciamiento correcto para la especie en estudio.

En el área experimental, las plantas fueron organizadas en tres bloques con $200 \mathrm{~m}^{2}$ cada uno, en los cuales se trasplantaron 100 plantas en cada bloque, totalizando 300 plantas. En el bloque tres fueron trasplantadas las mayores plantas - CDE 3, en el bloque dos las medianas - CDE 2 $y$ en el bloque uno las pequeñas - CDE 1. Las plantas se sistematizaron de acuerdo con la incidencia de vientos en el área, es decir, las plantas con el mayor diámetro del estípite se organizaron en el lugar con mayor acción del viento y las plantas con el menor diámetro del estípite, en el lugar con la menor acción del viento. Así, el tamaño de las plantas (circunferencia del diámetro del estípite) fue el factor de bloqueo.

En el proceso de plantación de las plantas, se realizó la apertura de cuevas de $50 \times 30 \mathrm{~cm}$, siendo el plantín cubier-
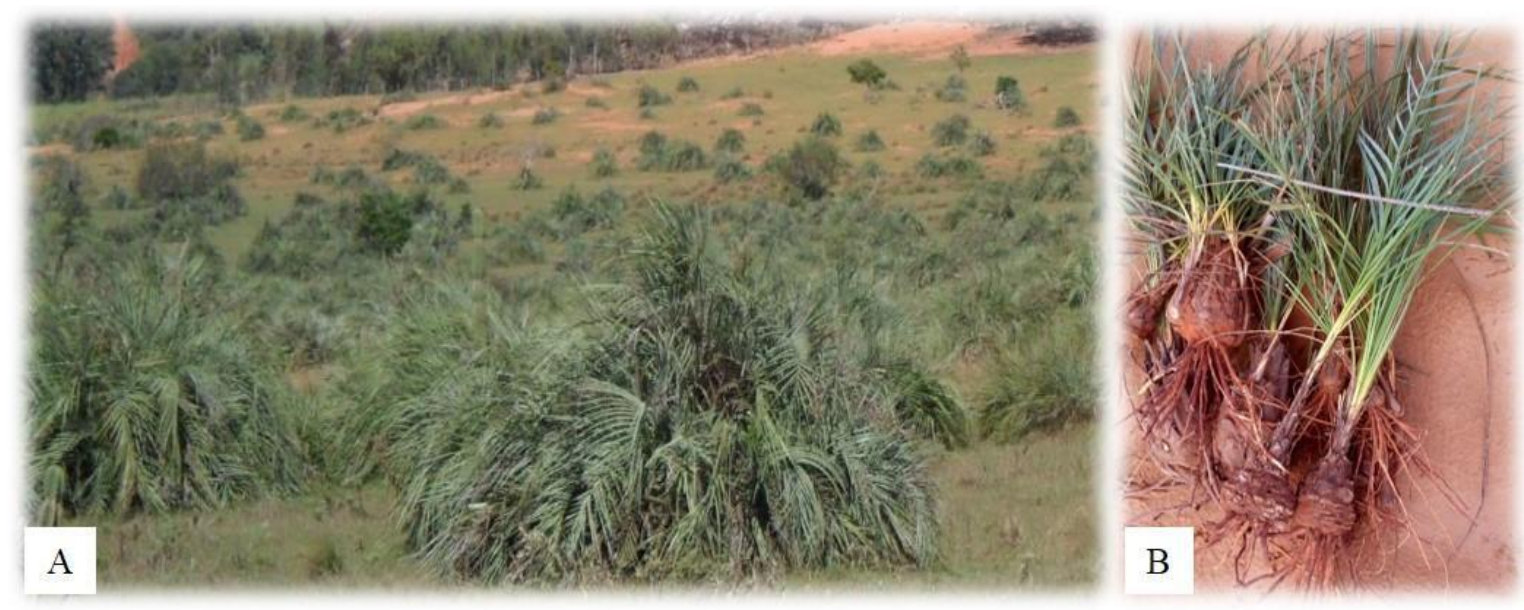

Figura 1. Población de plantas de Butia lallemantii a campo (A) y plantas individuales después de la recolección (B). Population of Butia lallemantii plants in the field (A) and individual molts after harvesting (B). 
ta con suelo hasta la altura del cuello (figura 2A). La disposición del hidrogel se dio por la mezcla del mismo al suelo utilizado en contacto con las raíces, mediante las dosis de 0 y 5 g por plantín (figura 2B). La aplicación del abono y del calcáreo se hizo en dos pequeñas cuevas, a unos $10 \mathrm{~cm}$ de distancia de cada plantín, siendo el calcáreo de un lado y el abono de otro (figura 2C). Después de la plantación, cada plantín fue irrigada con un litro de agua (figura 2D). En el curso del experimento, la irrigación de las plantas se sucedió de acuerdo con el régimen pluviométrico de la región, con mayor periodicidad en las estaciones de primaveraverano, ocurriendo en la primavera, especialmente los meses de febrero a marzo, con un promedio de $152 \mathrm{~mm}$, $\mathrm{y}$ en el verano, los meses de octubre a noviembre, con un promedio de 188,5 mm de lluvia, de acuerdo con Estación Meteorológica Cooperativa CAAL ubicada en el municipio de Alegrete-RS (CAAL 2015 y 2016).

Monitoreo del desplazamiento de arena en el área del estudio. La actividad intensiva de procesos erosivos en el área de estudio arenizada, principalmente, la constante movilidad de partículas de arena por la erosión eólica, justificó el monitoreo del movimiento de arena entre las plantas $B$. lallemantii y en entorno de los bloques. De esta forma, se sistematizaron cinco reglas de $110 \mathrm{~cm}$ de altura cada, en sus bloques, las cuales fueron introducidas en el suelo arenizado hasta los $60 \mathrm{~cm}$, para que sean realizadas las lecturas periódicas del nivel del suelo en relación al del inicio del experimento (figura 3 ).

Las variables analizadas fueron: la supervivencia, el aspecto foliar (número de hojas vivas por plantín) y alteración del nivel del suelo en función del desplazamiento de partículas de arena. Se consideraron sobrevivientes aquellas en las que el proceso de erosión eólica no ocasionó su completa remoción del suelo, ya que las plantas fuera del hoyo se consideraron muertas. El aspecto foliar fue evaluado visualmente, según la fisonomía de las plantas, siendo que las plantas con hojas verdes fueron consideradas con presencia de hojas vivas (PHV) y las plantas sin ninguna hoja verde fueron aquellas con ausencia de hojas vivas (AHV).

Análisis estadístico. El diseño experimental utilizado fue el de bloques aleatorizados, en los cuales el primer factor correspondió a las cinco dosis del abono mineral $(0,30$, 60,90 y 120 g por plantín) y el segundo factor las dosis del producto, hidrogel ( 0 y $5 \mathrm{~g}$ por plantín). Las plantas fueron sistematizadas en tres bloques, admitidos como factor de bloqueo el tamaño de las plantas (circunferencia del diámetro del estípite).

En el presente estudio, los resultados obtenidos se sometieron a la prueba de normalidad de Bartlett y, después

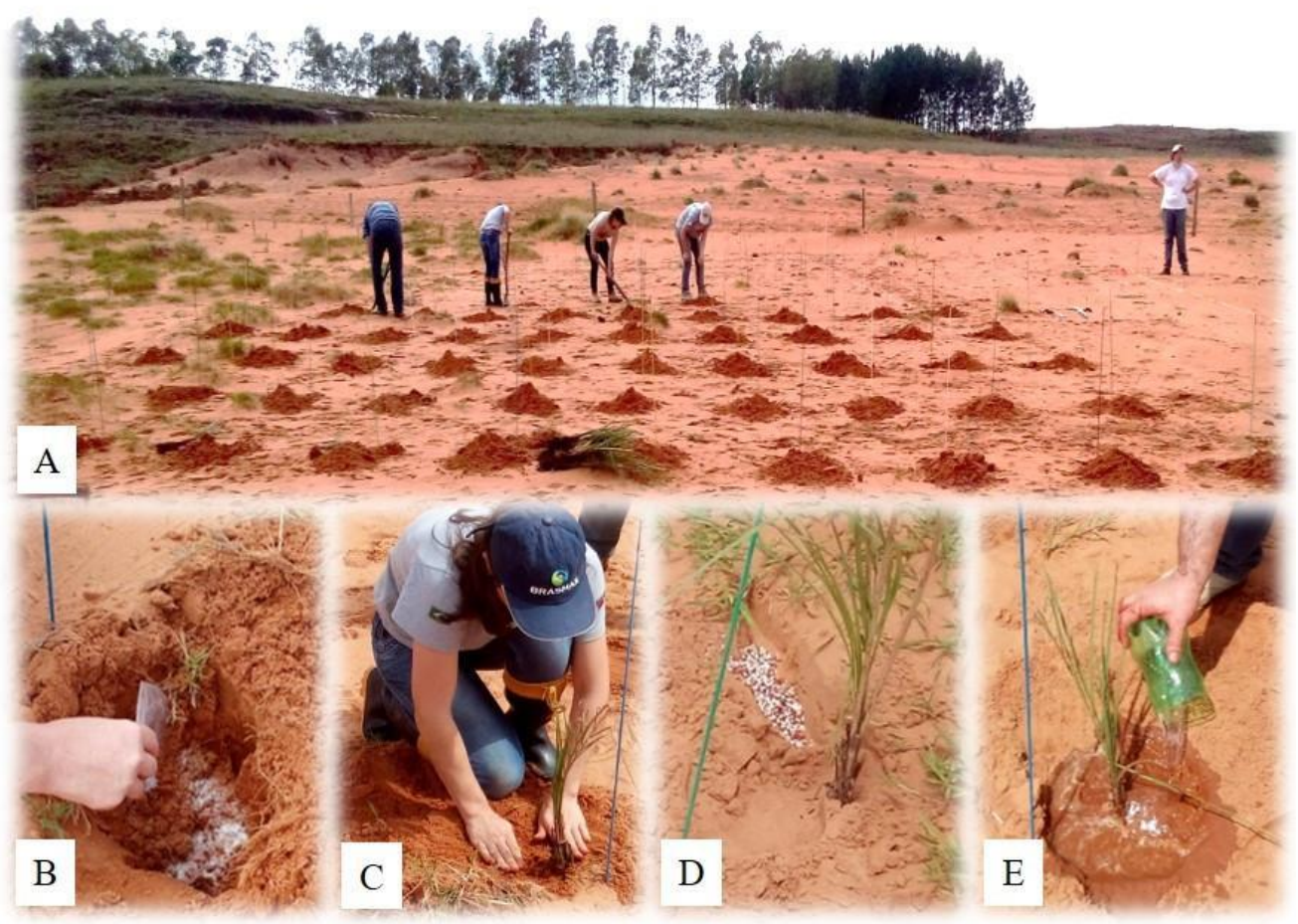

Figura 2. Plantación de las plantas de Butia lallemantii en área arenizada de estudio, por medio de los procedimientos de apertura de cuevas (A), aplicación de hidrogel (B), plantación (C), colocación de abono mineral (D), irrigación con un litro de agua (E).

Plantation of the wheels of Butia lallemantii in the sandstone area under study, through the procedures of opening caves (A), application of hydrogel (B), planting (C), placement of mineral fertilizer (D), irrigation with one liter of water (E). 
de cumplir con los supuestos del análisis de varianza, se sometieron a ANOVA y la comparación de medias por la prueba DMS (diferencia mínima significativa) al nivel de probabilidad del $5 \%$ de error, con la ayuda del software Costat 6.4.
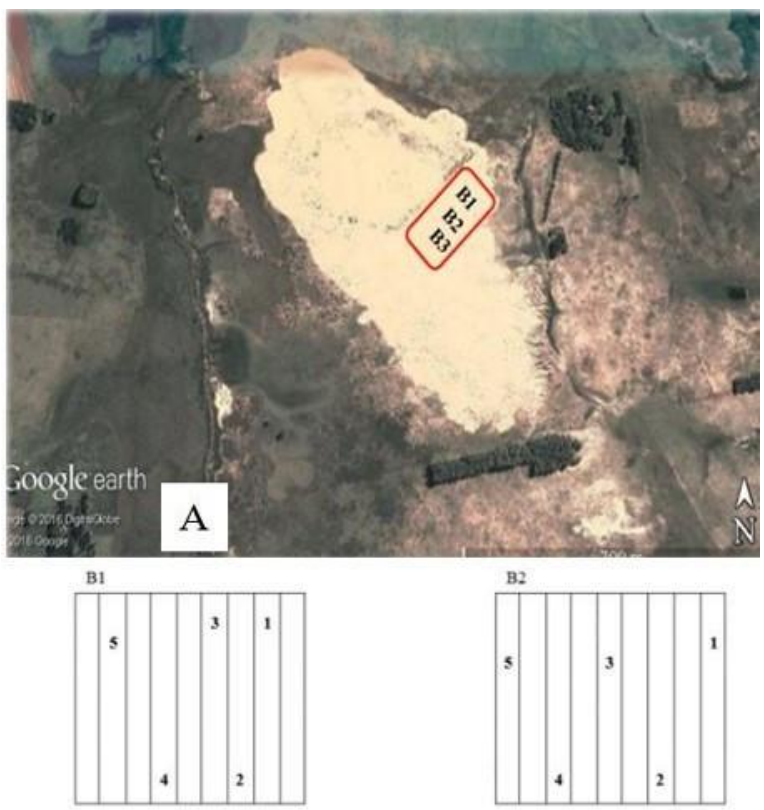

B
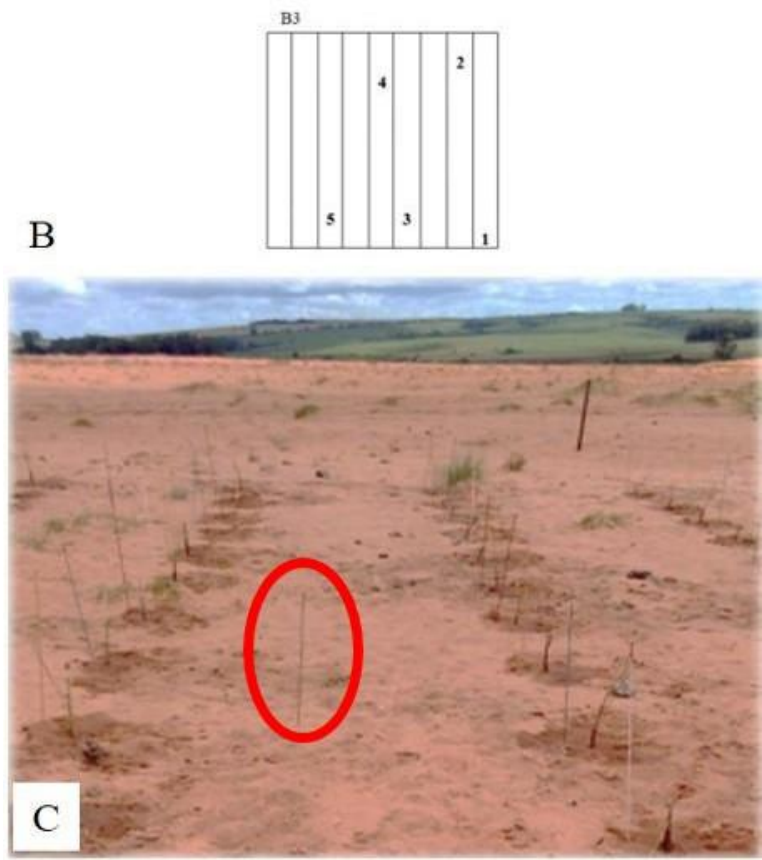

Figura 3. Posicionamiento de los bloques en el área experimental arenizada (A), distribución de las reglas en cada bloque (B) y demostración de reglas entre las plantas de Butia lallemantii a campo (C).

Positioning of the blocks in the experimental sandblasting area (A), distribution of the rules in each block (B) and the demonstration of the rules between the molts of Butia lallemantii on field (C).

\section{RESULTADOS}

El análisis estadístico no mostró diferencia significativa entre los niveles de cada factor (dosis de fertilización y de hidrogel), tampoco interacción entre ellos, sin embargo, hubo significancia entre los bloques para las variables presencia de hojas vivas y supervivencia de las plantas (cuadro 1). Las medias de estas variables fueron superiores en el bloque uno, con 39 y $97 \%$, respectivamente, siendo ese bloque el que presentaba las plantas más vigorosas y con mayor diámetro del estípite (cuadro 2).

Cuadro 1. Análisis de varianza para las variables presencia de hojas vivas (PHV), ausencia de hojas vivas (AHV) y supervivencia (SUP) de plantas de Butia lallemantii al final de 300 días, de acuerdo con tres bloques de diámetros de estípite (BDE), el uso del hidrogel y cinco dosis de fertilización química.

Analysis of variance for the variables presence of living leaves (PLL), absence of living leaves (ALL) and survival (SUR) of Butia lallemantii molts at the end of 300 days, according to the three blocks of diameters of stipe (BDS), the use of hydrogel and five doses of chemical fertilization.

\begin{tabular}{lccc}
\hline \multirow{2}{*}{ Factor } & \multicolumn{3}{c}{ Valor $P$} \\
\cline { 2 - 4 } & PHV (\%) & AHV (\%) & SUP (\%) \\
\hline Hidrogel & $0,101^{\mathrm{ns}}$ & $0,518^{\mathrm{ns}}$ & $0,052^{\mathrm{ns}}$ \\
Fertilización & $0,813^{\mathrm{ns}}$ & $0,215^{\mathrm{ns}}$ & $0,641^{\mathrm{ns}}$ \\
Interacción & $0,693^{\mathrm{ns}}$ & $0,555^{\mathrm{ns}}$ & $0,841^{\mathrm{ns}}$ \\
BDE & $<0,001$ & $0,123^{\mathrm{ns}}$ & $<0,001$ \\
\hline CV $(\%)$ & 89 & 34 & 26 \\
\hline
\end{tabular}

${ }^{n s}$ no significativo para el $5 \%$ de probabilidad de error; $\mathrm{CV}=$ coeficiente de variación.

Cuadro 2. Porcentaje promedio de presencia de hojas vivas (PHV) y de supervivencia (SUP) de plantas de Butia lallemantii al final de 300 días, de acuerdo con los tres bloques de diámetros de estípite (BDE 1: menores de 4,2 cm; BDE 2: 4,21 a 6,4 cm e BDE 3: 6,41 a más de $9 \mathrm{~cm}$ ).

Average data of the percentage of presence of living leaves (PLL) and survival (SUR) of the molts of Butia lallemantii at the end of 300 days, according to the three blocks of diameter of stipe (BDS 1: under $4.2 \mathrm{~cm}$; BDS 2: 4.21 to $6.4 \mathrm{~cm}$ and BDS 3: 6.41 to less than $9 \mathrm{~cm}$ ).

\begin{tabular}{ccc}
\hline BDE & PHV (\%) & SUP (\%) \\
\hline 1 & $39 \mathrm{a}$ & $97 \mathrm{a}$ \\
2 & $10 \mathrm{~b}$ & $76 \mathrm{~b}$ \\
3 & $3 \mathrm{~b}$ & $56 \mathrm{c}$ \\
\hline $\mathrm{CV}(\%)$ & 89,01 & 25,5 \\
\hline
\end{tabular}

$\mathrm{CV}=$ coeficiente de variación. En la columna, las medias seguidas por las mismas letras no difieren entre sí, por la prueba del DMS (5\%). 
La intensidad del desplazamiento de partículas de arena fue en promedio de dos a cuatro centímetros en el área de estudio, alcanzando especialmente el bloque uno. Además, no hubo diferencia significativa para el desplazamiento medio de arena en las cuatro estaciones del año, evidenciando apenas el desplazamiento alto ocurrió en el período de primavera (figura 4). En el primer bloque se observó un mayor desplazamiento de partículas de arena, especialmente en los meses que cubren la estación de primavera, con una media de aproximadamente cuatro centímetros de cambio de la capa de arena (figura 4).

En el bloque dos también sufrió desplazamiento de partículas de arena durante el período de la estación de primavera, en comparación con los demás, alcanzando un valor máximo de 2,5 cm de altura. En comparación con los bloques uno y dos, en el bloque tres se observaron resultados similares en el área a lo largo de las cuatro estaciones (figura 4). En ese bloque, el desplazamiento de arena fue subiendo al inicio del otoño.

\section{DISCUSIÓN}

Establecimiento de las plantas de Butia lallemantii en el área de estudio. El tallo de las palmeras, denominado estípite, puede presentar en su composición fibras, proteínas y polisacáridos (celulosa), los cuales propiciarán su forma y la presencia de material nutritivo. De esta forma, la acumulación de reservas en la base del estípite es de extrema importancia, pues actuaría en la conducción de agua y nutrientes para toda la planta, además, conforme Nair (2010) tiene la función de permitir mayor estabilidad contra la acción de vientos fuerte. En las palmeras con hábito de estípite cespitoso, como B. lallemantii, se forman plantas por la emisión de perfiles, con los cuales garantizan una mayor sostenibilidad y reserva de nutrientes para el desarrollo de las plantas (Nair 2010, Soares et al. 2014). En ese sentido, palmeras compuestas por estructuras de estípite cespitoso y de mayor diámetro demuestran alta relevancia para el buen desarrollo de las plantas, y consecuentemente supervivencia. Así como Paim y Paim (2016) verificaron elevada supervivencia de plantas de $B$. lallemantii trasplantadas a local en proceso de arenización, cuando utilizaron plantas con mayor tamaño de diámetro del estípite.

La restauración ecológica tiene como objetivo restaurar los procesos ecológicos perdidos de los ambientes degradados, y puede ser a través de la reintroducción de especies nativas, combinadas con técnicas de manejo, para permitir condiciones adecuadas de adaptación y perpetuación futura de las plantas. El uso del hidrogel en proyectos de restauración es de gran relevancia, pues de acuerdo con Cámara et al. (2011) son productos que presentan propiedades de retener y poner a disposición agua y nutrientes para las plantas por períodos prolongados. Los polímeros hidroretentores se incorporan en los sustratos en producción de plantas por disponer de agua y nutrientes lentamente para las plantas y minimizan los efectos de períodos caracterizados por alto calor en la fase de implantación de las plantas, favoreciendo el desarrollo en lugares más áridos (Cámara et al. 2011, Bernardi et al. 2012). Sin embargo, en el presente estudio, el análisis de varianza no evidenció resultados significativos del hidrogel para las variables de supervivencia y presencia de hojas vivas.

Posiblemente, la no influencia del hidrogel se explique por ser una especie nativa que se encuentra conspicuamente en campos arenosos y en las elevaciones areníticas de algunos municipios de la región oeste y suroeste del RS (Soares et al. 2014). En ese sentido, B. lallemantii presen-

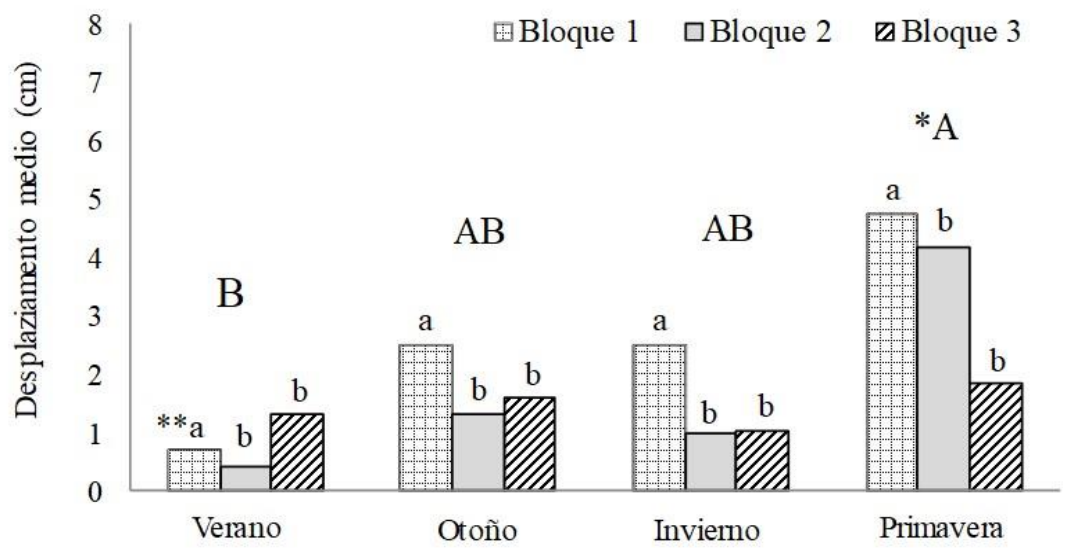

Figura 4. Cambio en el nivel del suelo debido al desplazamiento medio de arena $(\mathrm{cm})$ en área arenizada en los bloques uno, dos y tres, de acuerdo con las estaciones del año (* medias de las estaciones del año seguidas de letras iguales mayúsculas y ** las medias seguidas de letras minúsculas entre los bloques dentro de cada estación, no difieren entre sí por la prueba Duncan $5 \%$ ).

Change in soil level due to average displacement of sand $(\mathrm{cm})$ in a sandstone area in blocks one, two and three, according to the seasons of the year (*averages of the seasons of the year followed by equal capital letters and **the means followed by lowercase letters between the blocks within each station, do not differ from each other by the Duncan $5 \%$ test). 
ta características singulares de adaptabilidad y rusticidad que son favorables en el restablecimiento de la cobertura vegetal de locales arenizados. Según Suertegaray (2011) y König et al. (2014), los arenales se caracterizan como áreas de suelo expuesto, con baja disponibilidad hídrica y escasez de nutrientes, así como un reducido grado de agregación de partículas, en el que su constante movilidad dificulta la fijación de especies vegetales.

Las plantas de $B$. lallemantii transplantadas para los bloques dos y tres demostraron comportamiento análogo entre ellas, en función del reducido porte de diámetro del estípite de las plantas, las cuales presentaban baja fuente de reservas nutricionales y capacidad para tolerar la acción de procesos erosivos (eólicos y agua). Además, la acción del agente erosivo hídrico puede haber sido perjudicial para la conservación de las hojas vivas en estas plantas, ya que hubo restricción de los efectos de las dosis de fertilización química y del hidrogel. Este resultado puede estar relacionado con la ubicación del área de estudio y la mínima cobertura vegetal, las cuales propicien negativamente la elevada incidencia de vientos y lluvia (impactos de las gotas).

La introducción de plantas en suelos de textura arenosa presenta obstáculos, pues de acuerdo con König et al. (2014) en estas situaciones hay baja capacidad de retención de agua y restricción de la cantidad de nutrientes (materia orgánica y capacidad de intercambio catiónico), especialmente en gran parte de los locales arenosos del bioma Pampa. En cuanto a los locales arenizados estos aspectos se vuelven alarmantes, pues conforme Freitas et al. (2009) son suelos con bajísimo contenido de materia orgánica $(0,1$ a $0,7 \%$ ) y arcilla (6 a $12 \%)$, dando mínima resistencia a los agentes erosivos y, principalmente, baja capacidad de retención de nutrientes y almacenamiento de agua. Los mismos autores rectifican la fragilidad del sustrato de esas áreas y sus sedimentos poco consolidados, como verificados en el municipio de São Francisco de Assis/RS, cuyas características de menor saturación de bases, capacidad de intercambio catiónico, materia orgánica y contenido de nutrientes dificultaron el desarrollo de especies, resultando en plantas con mayor sensibilidad a los efectos de lixiviación.

Las dosis de fertilizante mineral utilizadas en este estudio no favorecieron la calidad visual de las palmas de $B$. lallemantii, lo que puede haber ocurrido debido al corto período de evaluación, asociado con el lento desarrollo de la especie. En la mayoría de los casos, el uso de fertilizantes químicos en el cultivo de palmeras no ha mostrado respuestas significativas en su desarrollo, como se puede ver en estudios con las especies de Rhapis excelsa (Thunb.) A. Henry ex Rehder]) (Luz et al. 2011) y Phoenix roebelenii O'Brien (Wanderley et al. 2012).

La práctica de fertilización asociada a técnicas de manejo puede posibilitar condiciones de cultivo más adecuadas, de acuerdo con Bernardi et al. (2012), que favorecen el desarrollo de las plantas, acelerando el crecimiento y las exigencias nutricionales. En este contexto, se destaca la importancia de trabajos que involucran conocimientos técnicos y fisiológicos sobre la eficacia del hidrogel asociado a la fertilización en la producción de especies nativas (Mews et al. 2015), especialmente aquellas con potencial para el restablecimiento de áreas degradadas.

Monitoreo del desplazamiento de arena en el área del estudio. La elevada intensidad en el desplazamiento de partículas de arena durante el período de primavera, puede haber sido en función de la declividad en torno del 3 $\%$, lo que evidencia un relieve plano a suave ondulado, con predominio de los vientos de noreste a suroeste, alcanzando principalmente el bloque 1, el cual se sitúa cerca del borde del arenal al inicio del área de estudio. Rovedder y Eltz (2008b) todavía resaltan que, el mayor desplazamiento sobre las áreas arenizadas ocurre sobre los meses de primavera-verano, que corresponden a los meses de mayor velocidad de los vientos en la región.

El bloque 1 se localiza en la región inicial del campo de arena y del área de estudio, siendo propenso la mayor incidencia de erosión eólica, de esa forma, en ese bloque se establecieron las plantas de $B$. lallemantii con mayor diámetro de estípite. El hecho de que la mayor supervivencia de las plantas haya sido observada en ese bloque, aun habiendo ocurrido la erosión eólica más intensa entre ellos, permite inferir que estas plantas presentan porte y condición de desarrollo más indicada para esa práctica. Soares et al. (2014) mencionan que el B. lallemantii presenta adaptabilidad a ambientes del tipo campestre y de áreas abiertas soleadas, las cuales están compuestas por suelos arenosos pobres en nutrientes y disponibilidad de agua.

En las áreas afectadas por el fenómeno de arenización ocurren procesos de retrabajo de los sedimentos areníticos debido a la elevada intensidad de los eventos erosivos hídrico y eólico, los cuales exponen esos suelos y perjudican la permanencia de especies vegetales (Rovedder et al. 2010, Suertegaray 2011). La erosión eólica dificulta el crecimiento y puede propiciar incluso la muerte de plantas, especialmente de porte herbáceo, pues ocasiona un efecto abrasivo de las partículas de arena en desplazamiento sobre las plantas, exponen las raíces por la supresión del suelo y ocurre el soterramiento en la fase de la deposición de las partículas (Rovedder y Eltz 2008b).

Rovedder y Eltz (2008a) observaron en área experimental compuesta por suelo arenizado que en los meses de primavera-verano hubo elevada intensidad de vientos, a pesar del empleo de plantas de cobertura como la Avena strigosa Schreb. y Lupinus albescens Hook. et Arn. El aumento de la dinámica y evolución de los arenales ocurre por el proceso de desplazamiento de partículas de arena en las capas superficiales del suelo por las erosiones eólica e hídrica, las cuales perjudican el establecimiento de especie vegetales sobre los Neosolos Quartzarénicos Órticos y manchas arenosas (Suertegaray y Oliveira 2014).

En este contexto, la especie de B. lallemantii solo se describió en 2006 como una planta endémica del bioma de 
Pampa en el estado de RS (Deble y Marchiori 2006). Merece destacarse por su potencial, desde la reconstitución de las áreas afectadas por el proceso de arenización hasta la explotación de sus frutos y hojas (alimentos y productos artesanales). Por lo tanto, el uso de especies nativas del sitio a recuperar se convierte en una buena alternativa para acelerar el proceso de restauración de áreas degradadas y el mantenimiento de la biodiversidad ambiental. De acuerdo con Schneider et al. (2014), este tipo de planta presenta una gran rusticidad y agresividad para competir con otras especies y proteger el suelo rápidamente.

Por lo tanto, es extremadamente importante alentar estudios científicos que aborden el uso de tecnologías asociadas con el régimen de prácticas fitotécnicas (fertilización mineral y/u orgánica, alternativas de riego, manejo de plantas, materiales biodegradables, entre otros), con el objetivo de favorecer el establecimiento y la longevidad de las especies de palmeras nativas en áreas afectadas por el fenómeno de arenización en el suroeste de RS.

\section{CONCLUSIONES}

El establecimiento de plantas de Butia lallemantii por el método de revegetación en suelo arenizado no requiere el uso de fertilizantes minerales y polímeros hidroretentores, de acuerdo con las condiciones del estudio.

Sin embargo, las plantas de $B$. lallemantii con un diámetro del estípite mayor a $6,4 \mathrm{~cm}$ deben usarse para favorecer la supervivencia y la presencia de hojas vivas en las plantas de campo.

Además, el desplazamiento de partículas de arena en áreas adyacentes a individuos de $B$. lallemantii es mayor los meses de primavera.

\section{AGRADECIMIENTOS}

Capes (Coordinación de Perfeccionamiento de Personal de Nivel Superior), CNPq (Consejo de Desarrollo Científico y Tecnológico) y la FAPERGS (Fundación de Amparo a la Investigación del Estado de Rio Grande do Sul) que contribuyeron al desarrollo del trabajo.

\section{REFERENCIAS}

Bernardi MR, M Sperotto Junior, O Daniel, ACT Vitorino. 2012. Crescimento de mudas de Corymbia citriodora em função do uso de hidrogel e adubação. Revista Cerne 18(1): 67-74. DOI: 10.1590/S0104-77602012000100009

Binda ALB, R Verdum. 2015. Reflexões interpretativas sobre as manchas de areia do sudoeste do Rio Grande do Sul, Brasil: da desertificação à arenização. Boletim Goiano de Geografia 35 (2): 273-288. DOI: https://doi.org/10.5216/ bgg.v35i2.37431

Boldrini II, PMA Ferreira, BO Andrade, AA Schneider, RB Setubal, R Trevisan, EM Freitas. 2010. Bioma Pampa: diversidade florística e fisionômica. Brasil, Porto Alegre. Editora Pallotti. 64 p.
Cámara GR, EF Reis, GL Araujo, MM Cazotti, JD Junior. 2011. Avaliação do desenvolvimento do cafeeiro conilon robusta tropical mediante uso de polímeros hidroretentores e diferentes turnos de rega. Enciclopédia Biosfera 7 (13): 135-146.

Deble LP, JNC Marchiori. 2006. Butia lallemantii uma nova Arecaceae do Brasil. Balduinia (9): 1- 3.

CAAL (Cooperativa Agroindustrial Alegrete, BR). 2015-2016. Registro de precipitação, da Estação Meteorológica Cooperativa Agroindustrial Alegrete Ltda., BR 290 - Km 587, 2014. Disponible: http://www.caal.com.br/previsao-tempo/ registro-de-chuvas

Freitas EM, II Boldrini, SC Müller, R Verdum. 2009. Florística e fitossociologia da vegetação de um campo sujeito à arenização no sudoeste do Estado do Rio Grande do Sul, Brasil. Acta Botânica Brasileira 23(2): 414-426. DOI: 10.1590/ $\underline{\text { S0102-33062009000200013 }}$

König F, CEP Gonçalves, AR Aguiar, AC Silva. 2014. Bioma Pampa: Interações entre micro-organismos e espécies vegetais nativas. Revista de Ciências Agrárias 37(1): 3-9.

Luz PB, PDO Paiva, AR Tavares, S Kanashiro, FFA Aguiar. 2011. Efeito de diferentes substratos e adubação fosfatada no crescimento de mudas de Rhapis excelsa (Thunb.) A. Henry ex Rehder (Palmeira-ráfia). Revista Brasileira de Horticultura Ornamental 17 (1): 37-42. DOI: https://doi. org/10.14295/rbho.v17i1.715

Mews CL, JRL Sousa, GTOS Azevedo, AM Souza. 2015. Efeito do Hidrogel e Ureia na produção de mudas de Handroanthus ochraceus (Cham.) Mattos. Floresta e Ambiente 22(1): 107-116. DOI: 10.1590/2179-8087.080814

Nair KPP. 2010. The agronomy and economy of important three crops of the developing world. Estados Unidos, San Diego. Elsevier Science \& Technology Books. 351 p.

Paim LP, MP Paim. 2016. Uso de mudas de Butia lallemantii (Deble \& Marchiori) para recuperar áreas degradadas por arenização no município de Alegrete-RS. Revista Congrega 13: 984-994.

Pillar VDP, SC Muller, ZMS Castilhos, AV Jacques. 2009. Campos Sulinos: conservação e uso sustentável da biodiversidade. Brasil, Brasília. Ministério do Meio Ambiente. 403 p.

Rodrigues ET, PAM Leal, E Costa, TS Paula, VA Gomes. 2010. Produção de mudas de tomateiro em diferentes substratos e recipientes em ambiente protegido. Horticultura Brasileira 28: 483-488. DOI: 10.1590/S0102-05362010000400018

Rossato MS. 2011. Os climas do Rio Grande do Sul: variabilidade, tendências e tipologia. Tesis Geógrafo. Porto Alegre, Brasil. Faculdade de Geografia, Universidade Federal do Rio Grande do Sul, Porto Alegre. 253 p.

Rovedder AP, FLF Eltz. 2008a. Desenvolvimento do Pinus elliottii e do Eucalyptus tereticorni consorciado com plantas de cobertura, em solos degradados por arenização. Ciência Rural 8 (1): 84-89. DOI: https://doi.org/10.1590/S0103$\underline{84782008000100014}$

Rovedder AP, FLF Eltz. 2008b. Revegetação com plantas de cobertura em solos arenizados sob erosão eólica no Rio Grande do Sul. Revista Brasileira de Ciência do Solo 32 (1): 315-321. DOI: http://dx.doi.org/10.1590/S0100$\underline{06832008000100029}$

Rovedder APM, Eltz FLF, Drescher MS, Dorneles FO, Schenato RB. 2010. Espaçamento entre linhas e densidade de semeadura em revegetação com espécie de tremoço visando à recuperação de solo degradado. Ciência Rural 
40(9): 1955-1960. DOI: http://dx.doi.org/10.1590/S0103$\underline{84782010005000135}$

Schneider PR, LEG Elesbão, PSP Schneider, RV Longhi. 2014. Crescimento em volume de Pinus elliottii e Pinus taeda em áreas arenizadas e degradadas no oeste do Rio Grande do Sul. Ciência Rural 44(9): 1561-1567. DOI: https://doi. org/10.1590/0103-8478cr20130744

Soares KP, SJ Longhi, LW Neto, LC Assis. 2014. Palmeiras (Arecaceae) no Rio Grande do Sul, Brasil. Rodriguésia 65: 113-139. DOI: http://dx.doi.org/10.1590/S217578602014000100009

Suertegaray DMA. 2011. Erosão nos campos sulinos: arenização no sudoeste do Rio grande do Sul. Revista Brasileira de Geomorfologia 12(3): 61-74.

Suertegaray DMA, Oliveira MG. 2014. Uma análise comparativa do processo de arenização no Brasil: sudoeste do Rio Grande do Sul e sudoeste de Goiás. Investigaciones Geográficas 47: 19-34.

Trindade JPP, Quadros FLF, Pillar VDP. 2008. Vegetação campestre de areais do Sudoeste do Rio Grande do Sul sob pastejo e com exclusão de pastejo. Pesquisa Agropecuária Brasileira 43(6): 771-779. DOI: http://dx.doi.org/10.1590/ S0100-204X2008000600014

Tucci CAF, Lima HN, Lessa JF. 2009. Adubação nitrogenada na produção de mudas de mogno (Swietenia macrophylla King). Acta Amazônica 39(2): 289-294. DOI: http://dx.doi. org/10.1590/S0044-59672009000200007

Vieira CL, Verdum R. 2015. Arenização e erosão hídrica no Sudoeste do Rio Grande do Sul: análise dos agentes condicionantes e considerações básicas para intervenções mecânico-vegetativas. Revista de Geografia 32(1): 41-65.

Vieira MA, Pauletto EA. 2009. Avaliação de atributos físicos do substrato de casca de arroz (Oryza sativa L.) carbonizada e tratada com polímeros hidrofílicos sintéticos. Bioscience Journal 25 (1): 1-6.

Wanderley CS, RT Faria, MU Ventura. 2012. Adubação química, adubação orgânica e extrato pirolenhoso no desenvolvimento de mudas de palmeiras fênix (Phoenix roebelenii). Semina 33(6): 2233-2240. DOI: $10.5433 / 1679-0359.2012 \mathrm{v} 33 \mathrm{n}$ $\underline{6 p 2233}$ 
